\title{
Quizzes: Quiz Application Development Using Android-Based MIT APP Inventor Platform
}

\author{
Muhammad Zubair Asghar ${ }^{1}$ \\ Institute of Computing and \\ Information Technology \\ Gomal University, D.I.Khan, \\ Pakistan
}

\author{
Iqra Sana $^{2}$ \\ Institute of Computing and \\ Information Technology \\ Gomal University, D.I.Khan, \\ Pakistan
}

\author{
Khushboo Nasir ${ }^{3}$ \\ Institute of Computing and \\ Information Technology \\ Gomal University, D.I.Khan, \\ Pakistan
}

\author{
Hina Iqbal ${ }^{4}$ \\ Institute of Computing and \\ Information Technology \\ Gomal University, D.I.Khan, \\ Pakistan
}

\author{
Fazal Masud Kundi ${ }^{5}$ \\ Institute of Computing and \\ Information Technology \\ Gomal University, D.I.Khan, \\ Pakistan
}

\author{
Sadia Ismail ${ }^{6}$ \\ Institute of Computing and \\ Information Technology \\ Gomal University, D.I.Khan, \\ Pakistan
}

\begin{abstract}
This work deals with the development of Androidbased multiple-choice question examination system, namely: Quizzes. This application is developed for educational purposes, allowing the users to prepare the multiple choice questions for different examinations conducted on provincial and national level. The main goal of the application is to enable users to practice for subjective tests conducted for admissions and recruitment, with the focus on Computer Science field. This quiz application includes three main modules, namely (i) computer science, (ii) verbal, and (iii) analytical. The computer science and verbal modules contain various sub-categories. This quiz includes three functions: (i) Hint, (ii) Skip, and (iii) Pause/life-lines. These functions can be used only once by a user. It shows progress feedback during quiz play, and at the end, the app also shows the result.
\end{abstract}

Keywords-Quiz; Android; MIT App Inventor; Interviews and test preparation

\section{INTRODUCTION}

Development of Android-based Quiz application is mainly required by students and learners to prepare themselves for different examinations directly through Smart-Phones and tablets in hands. The main aim of this project is to facilitate students in learning, gaining and improving their knowledge skills. At the meantime, our app provides them fun so that the users can prepare for interviews, entrance tests or any other corresponding purposes in a fresh mood and can't get bored or frustrated due to the dullness of application. We designed the application to facilitate the users to be able to take short quizzes using portable devices such as smart phones and tablets.

Byers and Alnarp [1] proposed an Interactive Learning Expert System for the Quizzes. They. In [2], authors proposed multiple-choice based quiz application using QuickBasic and JavaScript. Finally, the accuracy of correct answers is displayed after calculation. This quiz provides users the feature of making their own quiz. The operational version of the afore-mentioned system is available at [3].
The Web-based expert system proposed by [4], is the Student Edition for learning and preparation. It is a multiple choice quiz system. After each and every question, five choices are given. Users can select a single choice at a time. After giving an answer to all of the questions, users will submit the answers, and then a result or progress report is displayed containing total number and accuracy of correct, incorrect and un-answered questions. Email facility enables the users to send email to: their own id, an instructor, TA, and others.

The Quiz Hub [5] is an online Interactive Learning Quiz Games, focused on facts. This quiz has many sub-categories. It provides many fields to users, students and learners for the learning purpose. The categories are Math facts, U.S. History, Multiply fractions, Vocabulary Quiz, Spelling Quiz Game, Physics, and others. It is not a multiple choice quiz; one has to select the matching pairs in this quiz. Android is rapidly getting famous day by day, and the number of its users are increasing with each passing day, because it is easy to access the necessary Android-based applications on smartphones and tablets. Therefore, we found this idea easy and time efficient to facilitate the users in this way without any difficulty. There are many online quiz applications available on the internet, but most of them are only for entertainment and fun. Moreover, if one is going to appear in any test or interview, then it is time-consuming for them to read the full books or articles related to specific fields for the preparation or revising their knowledge.

However, the most attractive feature of our app is that we take learning and fun side by side. Our app provides them the facility to revise their knowledge or to learn something advantageous at one place without wasting their time.

The objective of this project is to develop an Androidbased system with following features, namely: (i) Questions bank, (ii) Time frame, (iii) Life lines, (iv) Data Storage, and (v) Multimedia support (pictures, snapshots, tables). The objective of creating this Quiz app is to help the users to 
prepare for necessary educational purposes regarding Computer Science and IT field with an easy access to our app directly on their Android phones. Through our app, users can learn and prepare themselves for interviews, tests and exams on Android phones, and can also use this app for increasing their general knowledge about Computer Science, Verbal and Analytical, everywhere and anytime.

\section{Material we used is Window 10 Haier laptop, MIT}

App Inventor 2 software, Windroy, QMobile Noir LT700, and Nokia Smart Phone.

Although there are a number of web-based and Androidbased applications which are, one way or other related to quiz, there are only few that help in learning and contribute to the academic enhancement of the students. Most of the available applications are aiming at having a fun or entertainment. Among the many applications, we review some Web-based and Android based applications that are quite famous and are successful regarding the amount of players and downloads.

Computer General Knowledge Quiz section is a repository of Multiple Choice Question that makes you aware about evolving nature of the competitive examination; this quiz is about subjects related to the computer field. It's a general computer quiz. This quiz is useful for the preparation of any computer field test. In this quiz app, questions are given along with four choices, and at the end, the correct choice is also given. After preparation, students can check their level of preparation through the quiz [6].

It is a Computer Science Quiz. It contains multiple choice questions and answers with explanations and examples. Operating System, Database Management System, Software Engineering, Computer Networks, Digital Electronics are the sub-fields present in this quiz. These Computer Science MCQs will help users for various Interviews, competitive exams, entrance exams, and others [7].

TreeKnox Computer Quiz is a quiz system for the help and preparation of computer science and IT students who are going to appear in any interview, tests or exams in computer science and IT field. Questions are given along with multiple choices and at the end of each question; a button named "Answer" is given. On clicking that button the correct answer is highlighted at the mean time [8]. This quiz application is very simple and interactive. In this there are two modes General and Aptitude, after selecting one of them it will be redirected to the Quiz interface which will contain the question with multiple answers (options) and contain three buttons "Submit", "Show Answer" and "Next" [9].

It is also a simple and interactive application [10]. It contains three modes "Easy", "Normal" and "Hard". After selecting one of them it will be redirected to the Quiz interface which contains questions with two options, True " $T$ " and False "F". It also show hints when user wants but if the user will try to use this life line "Hint" more than one time then it will show the answer not hint. Thus, it is useless because user can't learn anything from it anymore.
Although there are many apps that focus on the quiz, there are limited applications with focus on learning or improving knowledge in the curriculum area. Most of the other apps are entertainment-based with little focus on the educational paradigm.

There are many limitations with the existing systems mentioned above. To overcome such limitations, we propose user-friendly application, namely "Quizzes," which mainly focuses on gaining the curriculum knowledge as well as entertainment. Therefore, where one is amazed at playing the quiz, he/she is gaining curriculum knowledge with emphasis on not only gaining good grades but also having a better understanding of the subject matter.

Another unique feature of Quizzes that is lacking in other apps is the life lines, which it provides to the user. Users can view the hints for the right answer, can skip a question and also pause the quiz app for thirty seconds. We provided the life lines for the particular questions or the category itself, but user can use these life lines only once.

Other features regarding Quizzes and other apps seemed to be quite similar, i.e. answering questions with multiple choices as fast as possible, scoring as high as possible among the group, and so on.

There are many systems on the quiz-related content analysis in the context of opinion mining and other disciplines of computer science $[11,12,13,14,15,16,17]$, however, most of such studies are web-based and address the user generated contents.

The quiz above are either web-based recommendation systems or intelligent expert systems. Therefore, there is a need to develop an Android-based easy to use application.

\section{Methodology}

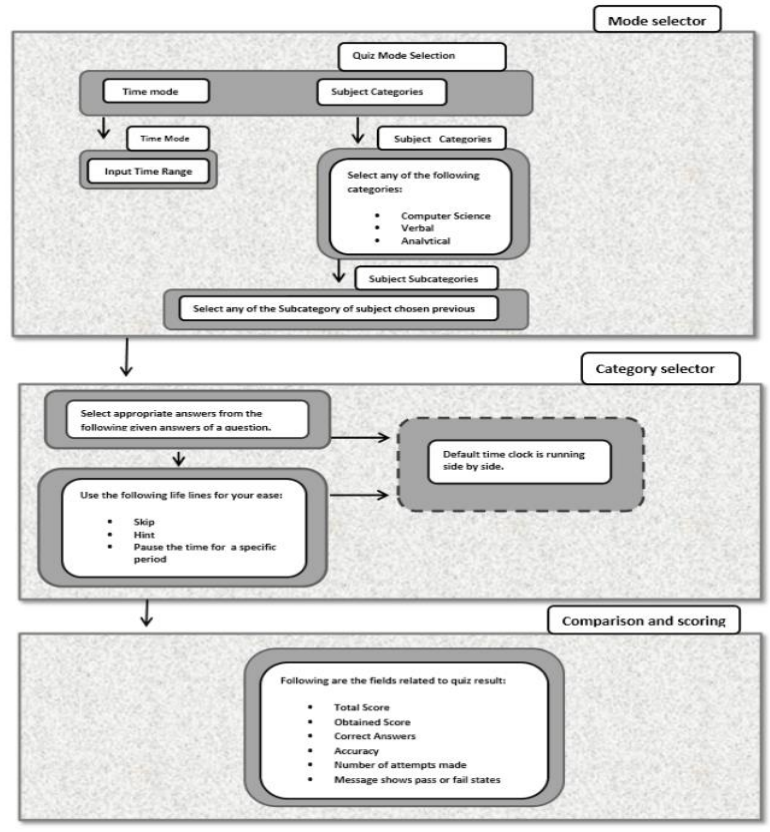

Fig. 1. The proposed system 
The proposed framework Fig. 1. comprises the following modules: (i) Mode selector, (ii) Category selector, and (iii) Comparison and Scoring.

\section{A. Mode Selector}

The mode selection module allows the user to select a mode out of given modes, that is, Time mode and Categories mode. If the user selects any mode out of these modes then he/she has to give certain inputs, like set the time range, selecting categories and sub-categories. The detailed flowchart of mode selection is presented in Fig. 2.

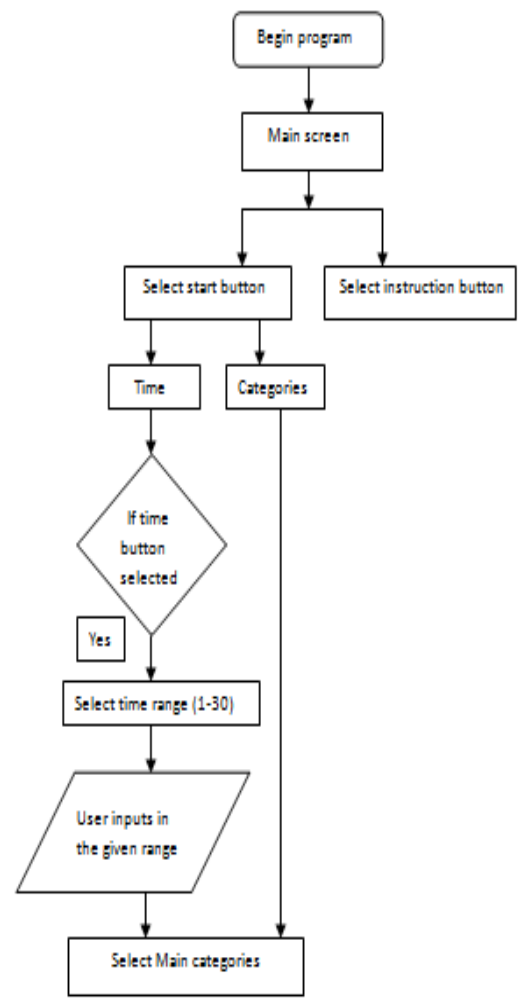

Fig. 2. Flow chart of mode selector

Algorithm.1 selecting the mode

Purpose: The purpose of this pseudo code is to select the mode. User can select any mode i.e. time or category.

Input: Time, Categories, Time Range

Output: time range, main categories

Begin

1. Lbtime $\leftarrow 0 / /$ initialize time range variable

2. If Mode $=$ Time Then

3. $\{$ Display time range $\}$

// $\ldots \ldots \ldots \ldots \ldots \ldots \ldots . . . .$.

4. If (time screen is initialized) then

5. $\{$ Set Listpicker.element $=1$ to 30
6. Set continuebutton.enabled $=$ false

7. Call DB.store value

8. IF (listpicker.afterpicking =true) then

9. $\{$ Set continuebutton.enabled $=$ true

10. Set $\mathrm{lb}$ time $=$ listpicker.selection $\}$

11. If listpicker.selection $<10$ then

12. $\{$ Append 0 ahead of Lbtime

13. Lbtime $=$ lbtime -1$\}$

14. If $($ Mode $=$ NonTime $)$ Then

15. Display main categories

16. End

\section{B. Category Selector}

In this module, the questions are given along with the multiple choices Fig. 3. The user has to give input by selecting any of the given options, and then these inputs are used in the calculation of final result. Some life lines are given to the user which assists to play the quiz more efficiently.

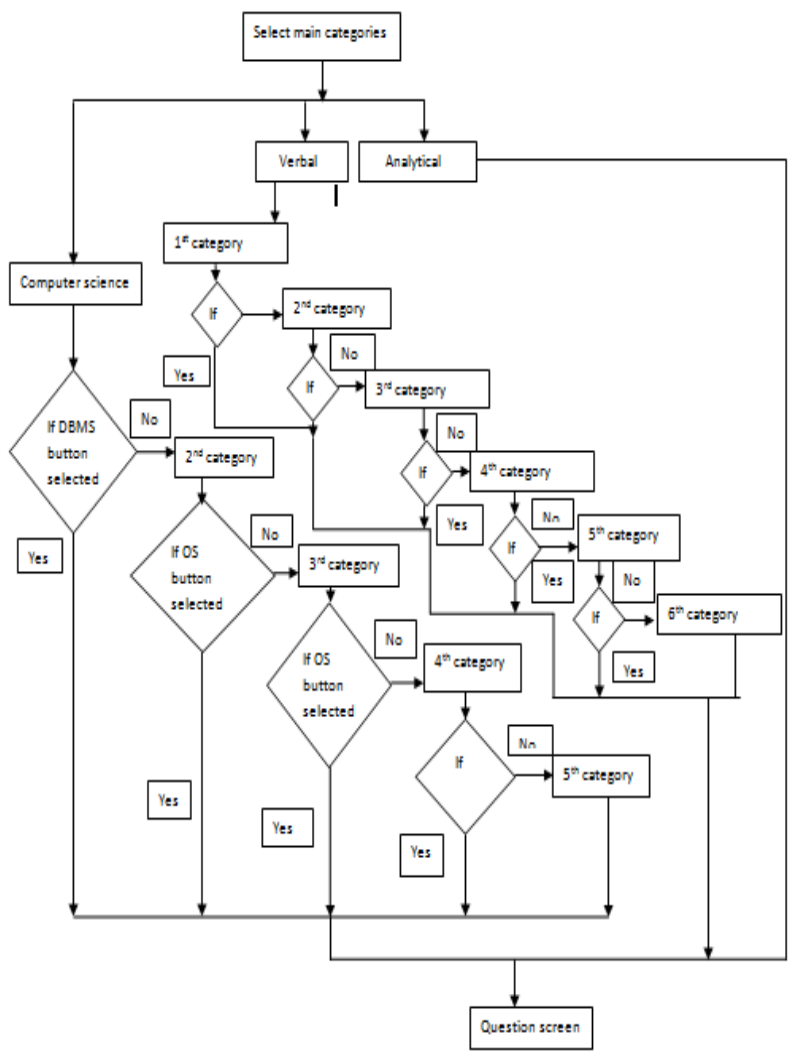

Fig. 3. Flow chart of category selector

Algorithm.2 selecting the main category

Purpose: The purpose of this pseudo code is to select the sub category of main category.

Input:Main_categories_list $=\{$ Computer, Verbal, Analytical $\}$, sub_categories_list $1=\{$ Operating system, 


\begin{abstract}
Database Management System, Computer Architecture, Data Structure, Computer Networking\}, sub_categories_list $2=\{$ Synonyms, Antonyms, Spellings, Ordering of words, Selecting words, Verbal Analogies\}
\end{abstract}

Output: Sub categories_list $1=\{$ Operating system, Database Management System, Computer Architecture, Data Structure, Computer Networking\}, sub_categories_list $2=\{$ Synonyms, Antonyms, Spellings, Ordering of words, Selecting words, Verbal Analogies\}, Question screen

\section{Begin}

\section{// Initialization}

1. Initialize sub categories \{Operating system, Database Management System,

Computer Architecture, Data Structure, Computer Networking, Synonyms, Antonyms, Spellings, Ordering of words, Selecting words, Verbal Analogies, Analytical \} by assigning the list of questions and option answers.

2. If category $=$ Computer Science Then

$3 . \quad\{$ Select any computer science's sub category from sub_categories_list1\}

4. If (any of the subjects from sub_categories_list1 is selected) Then

5. sub_categories_list1.selected

$\{$ Set "category" tag to

6. Display Question Screen $\}$

7. Else If (category $=$ Verbal ) Then

8. \{Select any Verbal's sub category fromsub_categories_list2\}

9. If any of the sub_categories_list2 is clicked Then

$10 . \quad\{$ Set "category" tag to sub_categories_list2.selected

\section{Display Question Screen}

12. Else category $=$ Analytical Then

13. Display Question screen

14. End if

End

\section{Comparison and Scoring}

Here, outputs such as total score, obtained score, accuracy, and number of attempts made are displayed as also the messages showing whether the users passed or failed the quiz, based on the given inputs provided by the users. The entire process is shown in Fig. 4.

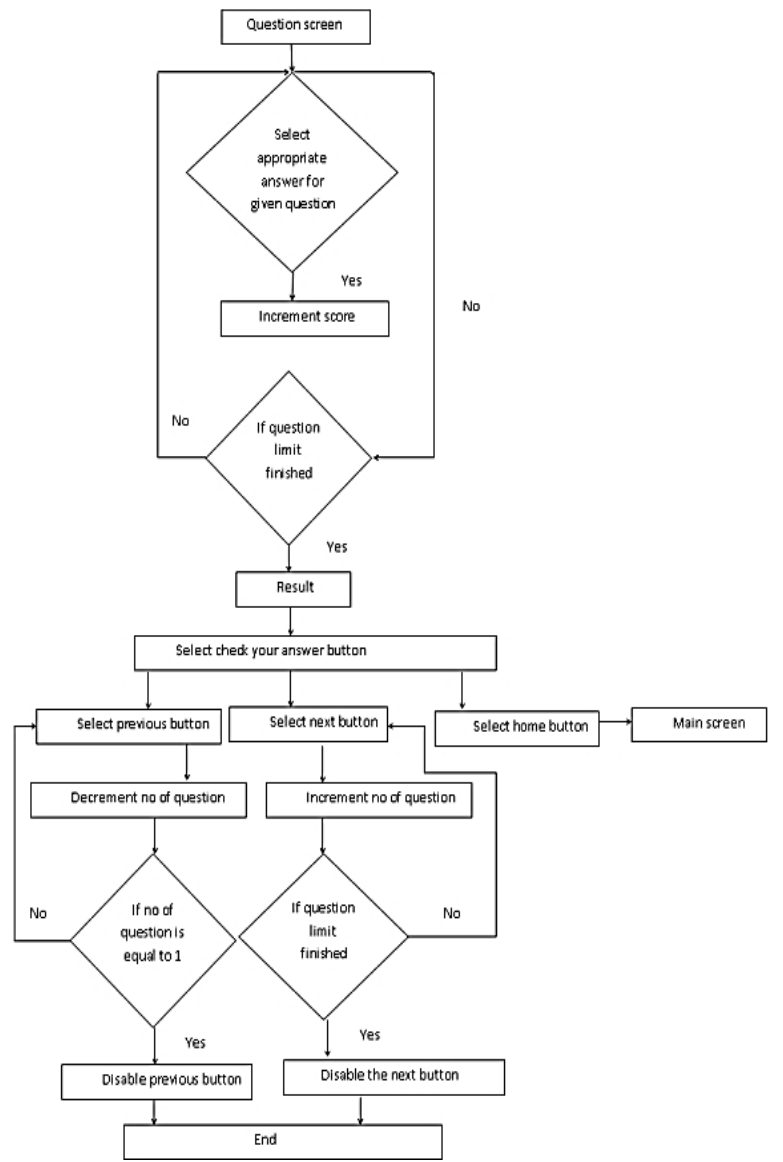

Fig. 4. Flow chart of comparison and scoring

Algorithm.3 Calculating the result and showing the right answer

Purpose: The purpose of this pseudo code is to show the appropriate answer of the question and to show the final results of the played quiz.

Input: appropriate answer for the given question

Output: result (total score, obtained score, correct answers, accuracy, number of attempts and message shows pass or fail states), answer key

\section{Begin:}

1. Do while (question limit is not finished)

2. Select appropriate answer for the given question

3. score $\leftarrow$ score +5

4. End while

5. Display the result screen

6. Select check your answer key

7. Select the previous button

8. (no_(of_questions_in_answer key) $\leftarrow$

(no_(of_questions_in_answer key) -1

9. If (No_of_questions_ in _ answer key $>1$ ) then

10. \{ Enable previous button

11. Else

12. Disable previous button

13. Or

14. Select next button 
15. (no_of_questions_in_answerkey) $\leftarrow$ (no_of_questions_ in_answerkey) +1

16. If (no_of_questions_limit_finished_answerkey) then

17. \{ Disable next button

18. Else

19. Enable next button

20. End if

End

\section{EXPERIMENTAL SETUP}

\section{A. Implementation}

A partial list of coding of proposed modules, namely (i) mode selector, (ii) category selector and (iii) comparison and scoring.

\section{Coding of mode selector module}

In Fig. 5, there are two button events namely (i) btnTime and (ii) btncategories, and there is a list picker. In these button event, there is tinyDB in which time and categories mode selection are save user can select any mode. In list picker IF THEN statement is used, and the user can select any choice from the list.
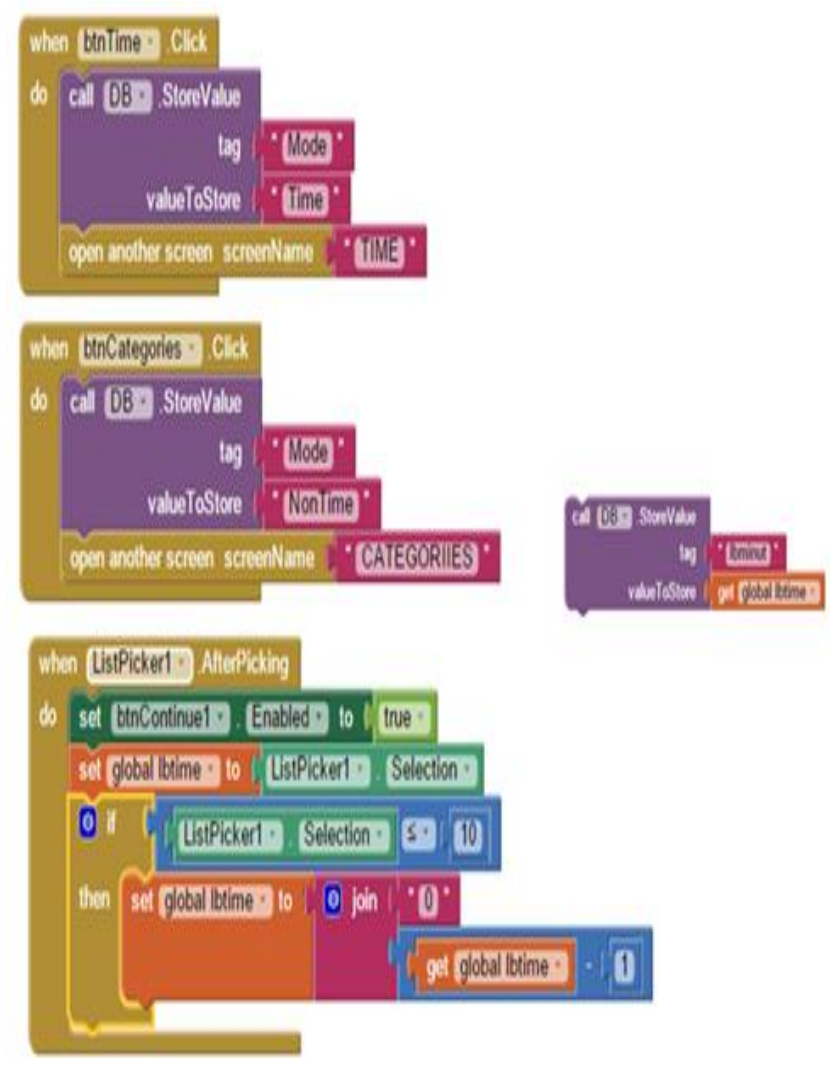

Fig. 5. Category selection and list picker

In Fig. 6, there is a list picker in which a list is made for selecting any time range. The continue button enabled property is made false.
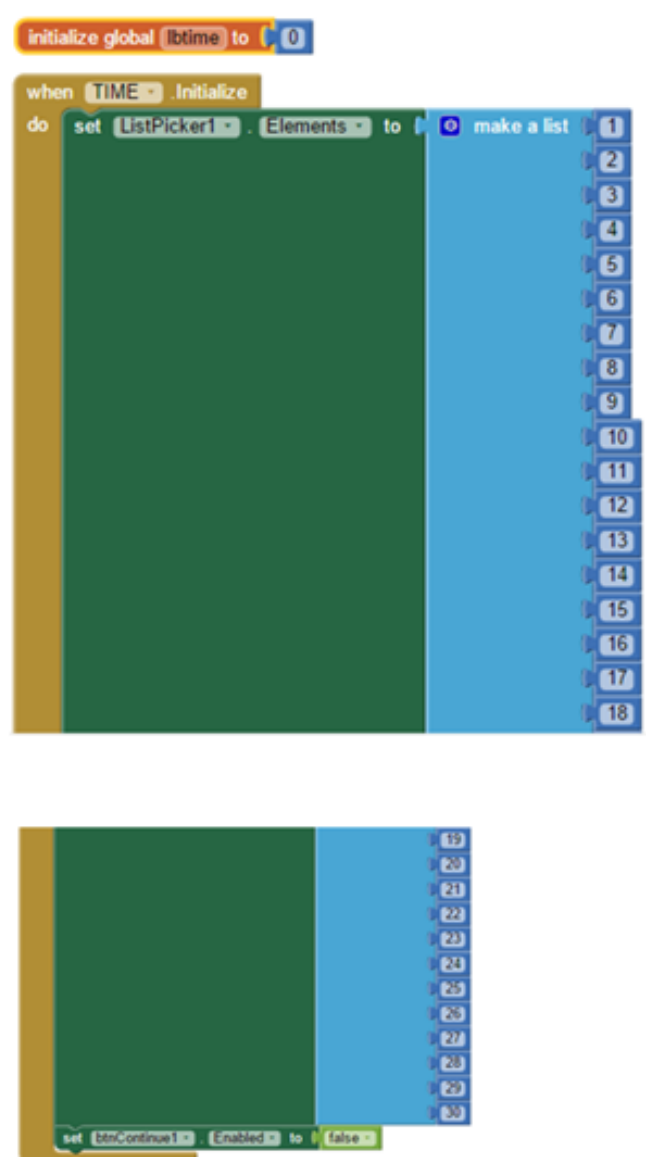

Fig. 6. Showing the list

In Fig. 7, there are five button events. In each button event, there is tinyDB; tinyDB is used to store the data and value. After selecting the desired category, the question screen will be opened.

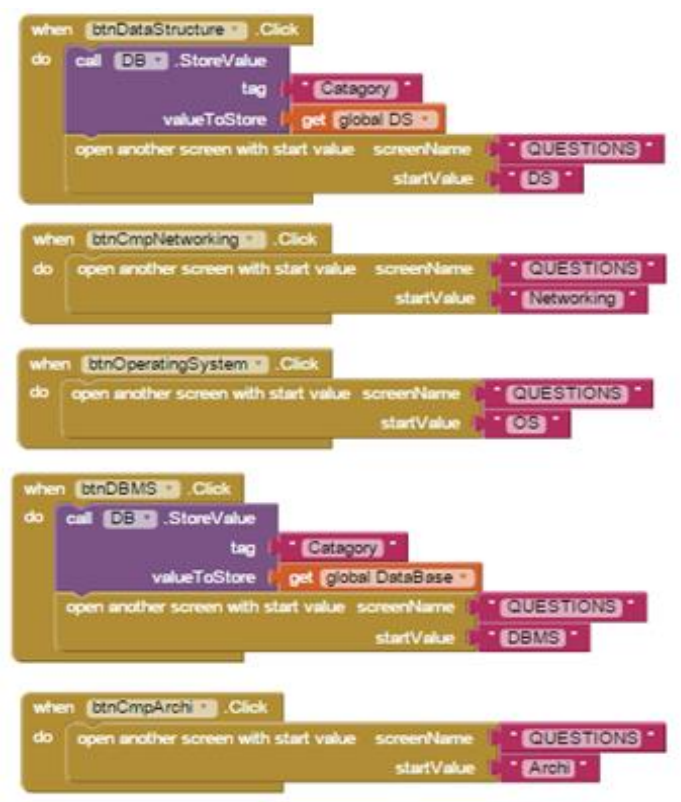

Fig. 7. Selecting sub-category and opening of question screen 
In Fig. 8, there are different sub-categories buttons namely

(i) BtnAntonyms (ii) BtnSpottingErrors

(iii) BtnComprehension (iv) BtnSynonyms

(v) BtnSelectingWords (vi) BtnSpelling

(vii) BtnVerbalAnalogies (vii) BtnOrderingOfWords

These buttons are event handlers. In this event handler, the TinyDB is used to store the desired questions of the all sub categories.
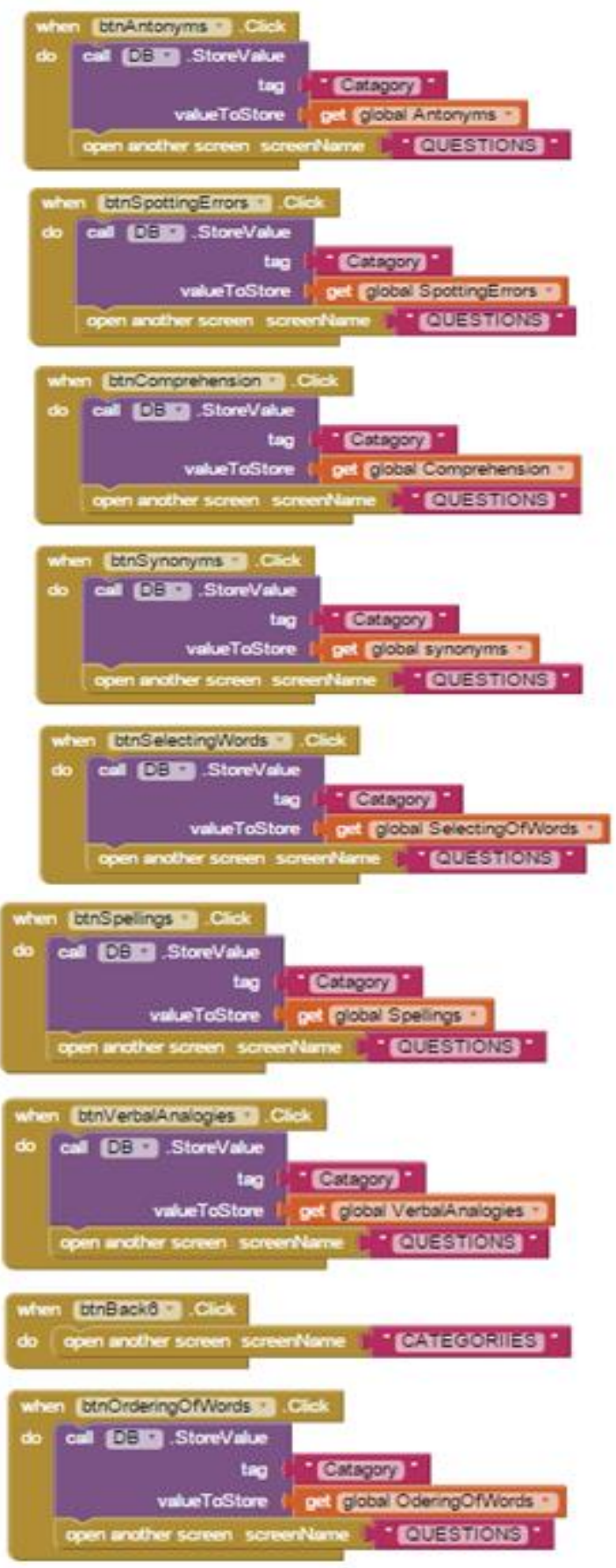

Fig. 8. Sub categories of main verbal category
In Fig. 9, the btnAnalytical is event handler in which the TinyDB is used to store the questions of the analytical category.

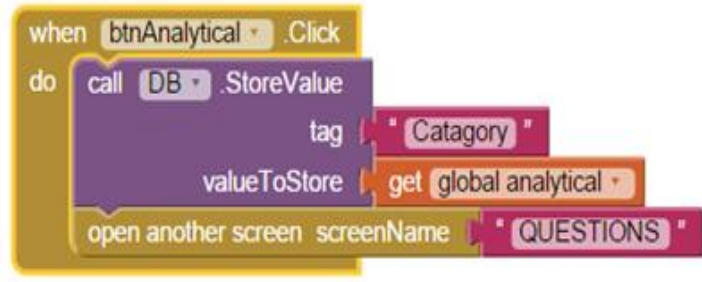

Fig. 9. Showing the question of analytical main category

\section{Coding of comparison and scoring module}

In Fig. 10, these blocks of code life line time are enabled as false when question is initializing and there are three procedures. First procedure calls for the time and question category, the second procedure calls for the next category, and the last procedure calls for the next question.

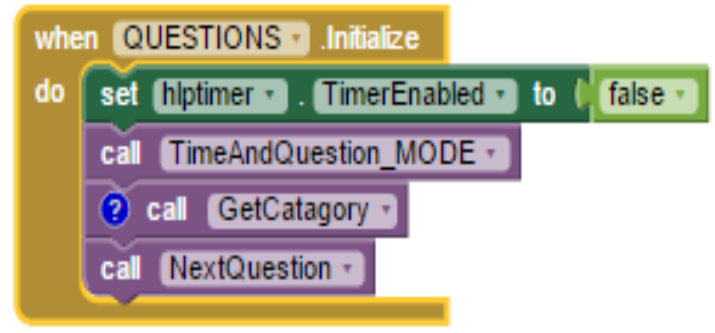

Fig. 10. Code blocks of life line

In Fig. 11, the procedure namely TimeAndQuestion_Mode is used in which IF THEN ELSE statement is used, TinyDB is also used and some textboxes and labels.

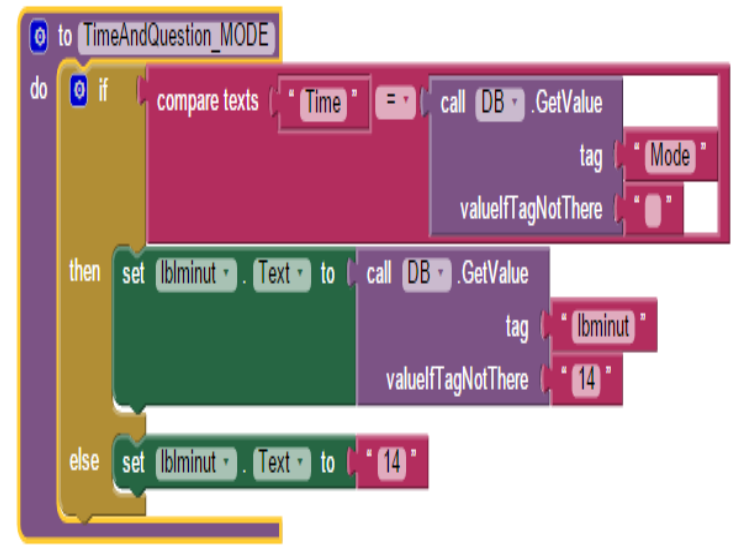

Fig. 11. Time and question mode

The procedure namely Final Result is used in which TinyDB is used nine times. IF THEN, ELSE IF THEN is used. All these instructions are executed in sequence. Set of labels and variables are also used and some of the blocks are dropped from the math Block editor. Fig. 12 shows the following code blocks. In final results, the results are calculated. 


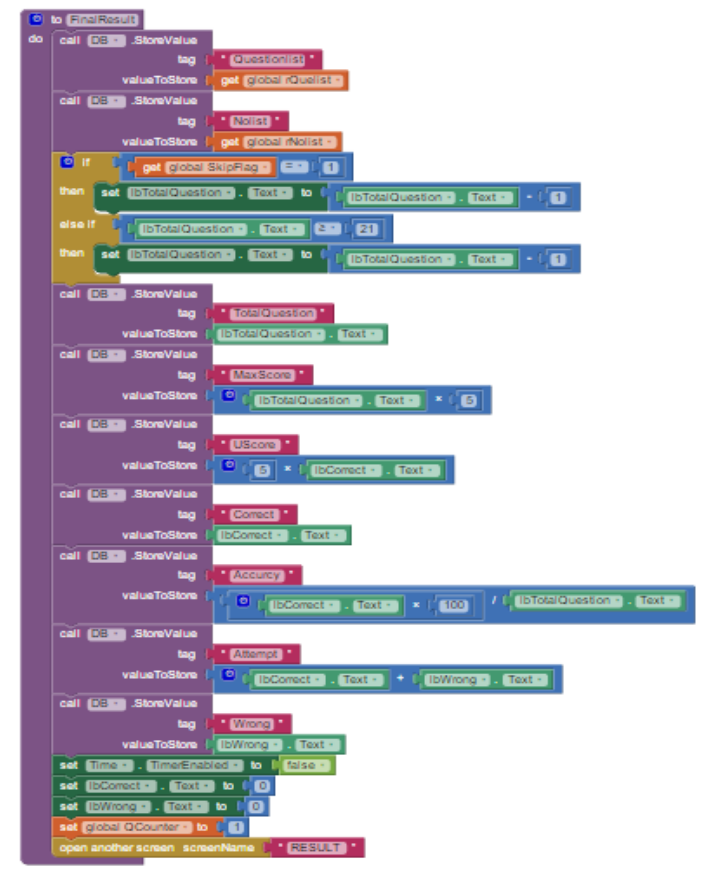

Fig. 12. Final result coding

\section{B. Results}

We executed our Quizzes application using Androidbased platform. Fig. 13 to 7 shows the output screens of the main application.
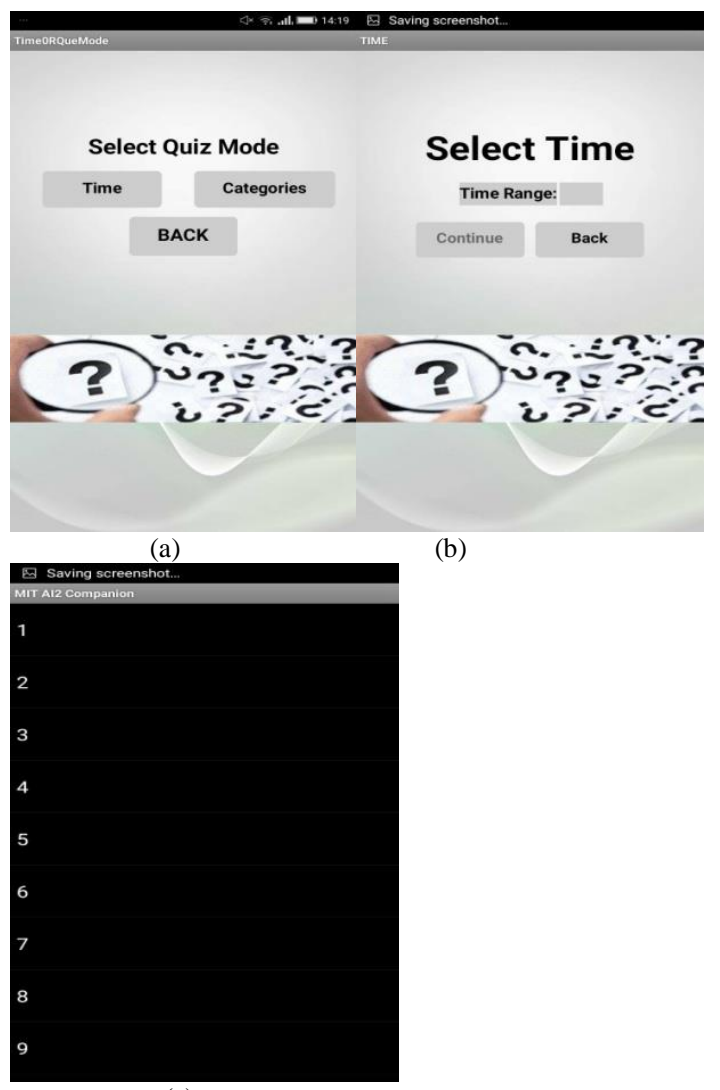

(b)

(c)

Fig. 13. (a) (b) (c) Quiz mode selection module

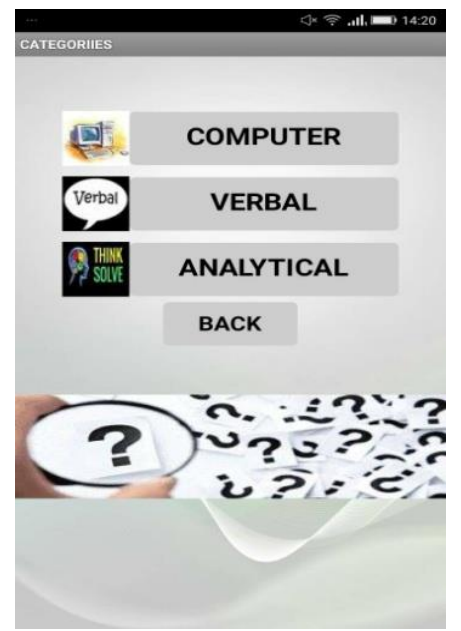

(a)

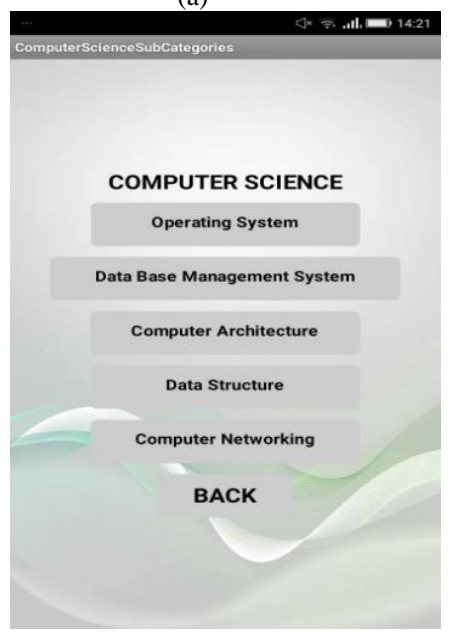

(b)

Fig. 14. (a) (b) Categories selection

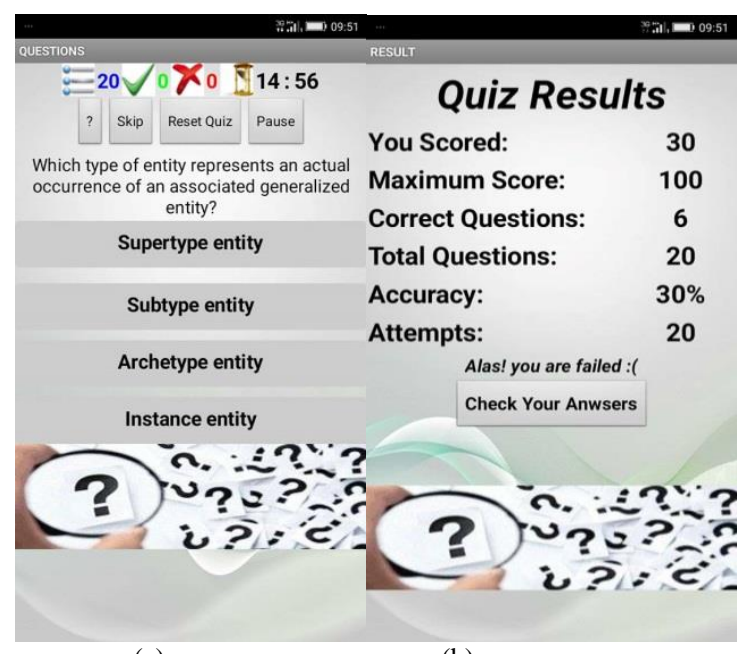

(a)

(b)

Fig. 15. Questions and Results (a) input (b) output

\section{Descriptive Analysis of data}

We asked some questions about our application to users and with the feedback we analysed our application. 


\section{1) Gender}

TABLE I. SHOWING THE BASIC STATISTICS OF GENDER

\begin{tabular}{|l|l|l|l|l|l|}
\hline \multirow{2}{*}{ Sr.no } & \multicolumn{4}{|c|}{ basic statistics } \\
\cline { 2 - 6 } & Minimum & maximum & Median & mean & standard deviation \\
\hline 1. & 1.00 & 2.00 & 2.00 & 1.56 & 0.50 \\
\hline
\end{tabular}

The minimum and maximum show the smallest and largest number answer choice that obtained minimum one response. It is beneficial to find the range of answers. The minimum and maximum of 1 and 2 show that there were 16 responses in the uppermost answer (i.e. Female) and 20 responses in the lower most answer (i.e. Male). The median represents the answer choice in the center of all your responses, that is, $50 \%$ below and $50 \%$ above the middle answer choice. The median of 2.00 (higher than the 1.56 mean) shows that there were more respondents who were male than the respondents who were female. A mean is the average of whole responses by adds up all the numbers and then divide them by total amount of number. A mean of 1.56 represents the overall respondents came in somewhere between male and female. Ultimately, the standard deviation is 0.50 which shows the progress, dispersion, and variation of your responses.

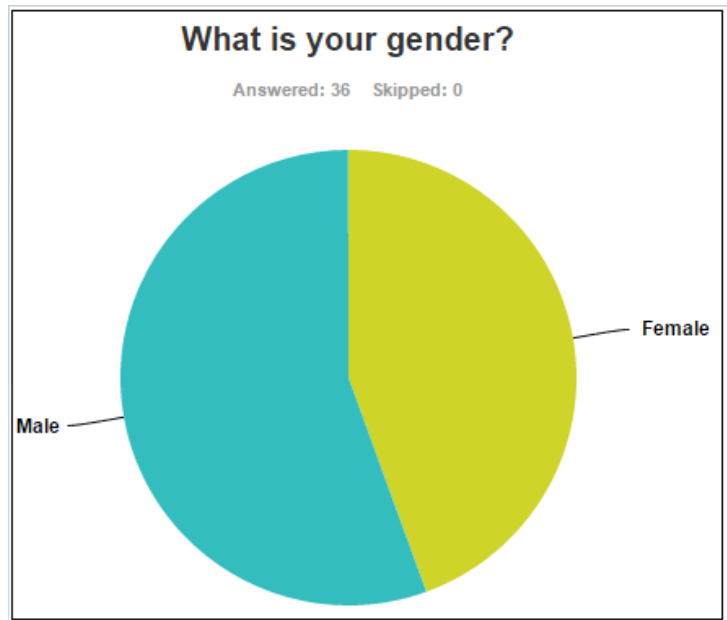

Fig. 16. Pie chart of gender

Fig. 16 shows the percentage distribution of the respondents according to gender: $44.44 \%$ of respondents were female, and $55.56 \%$ were male.

\section{2) Age}

TABLE II. Showing The BASIC Statistics of AgE

\begin{tabular}{|l|l|l|l|l|l|}
\hline \multirow{3}{*}{ Sr.no } & \multicolumn{5}{|c|}{ basic statistics } \\
\cline { 2 - 6 } & Minimum & maximum & Median & mean & standard deviation \\
\cline { 2 - 6 } 1. & 1.00 & 5.00 & 2.00 & 2.85 & 0.83 \\
\hline
\end{tabular}

The minimum and maximum of 1 and 5 show that there was one response in the uppermost answer( i.e. age 18 to 24) and one response in the lower most answer( i.e. age 55 to 64). The median of 2.00 (lower than the 2.58 mean) shows that there were more respondents whose age was between 25 and 34 than the respondents whose age was in between 35 and 44 . A mean of 2.58 represents that the overall respondents came in somewhere between 25 and 34 and 35 and 44. Ultimately, the standard deviation is 0.83 which shows the progress, dispersion, and variation of your responses.

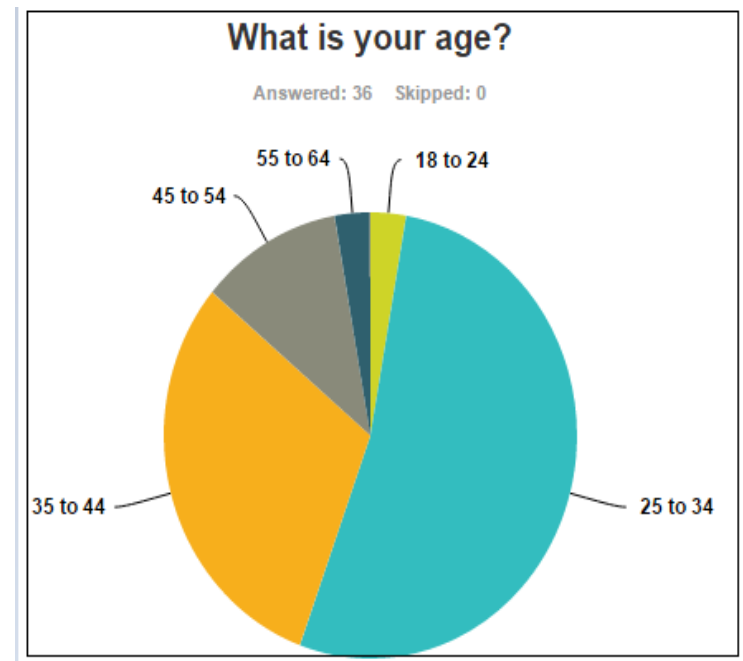

Fig. 17. Pie chart showing the age

Fig. 17 shows that there were $2.78 \%$ respondents whose age is between 18 and $24,52.78 \%$ respondents whose age is between 25 and 34, 30.56\% respondents whose age is between 35 and 44 and $2.78 \%$ respondents whose age is between 55 and 64 .

\section{3) Engaged in educational activities}

TABLE III. SHOWING THE BASIC STATISTICS OF ENGAGEMENT IN EDUCATIONAL ACTIVITIES

\begin{tabular}{|l|l|l|l|l|l|}
\hline \multirow{3}{*}{ Sr.no } & \multicolumn{5}{|c|}{ basic statistics } \\
\cline { 2 - 6 } & Minimum & maximum & Median & mean & standard deviation \\
\cline { 2 - 6 } 1. & 1.00 & 2.00 & 1.00 & 1.36 & 0.48 \\
\hline
\end{tabular}

The minimum and maximum of 1 and 4 shows that there were six responses in the uppermost answer (i.e. once in a while) and six responses in the lower most answer (i.e. almost never). The median of 2.00 (lower than the 2.29 mean) shows that there were more responses which were engaged with this app sometimes than the responses who were engaged almost all the time with the app. A mean of 2.29 represents the overall respondents came in somewhere between Sometimes or Once in a while. Ultimately, the standard deviation is 0.94 which shows the progress, dispersion, and variation of responses. 


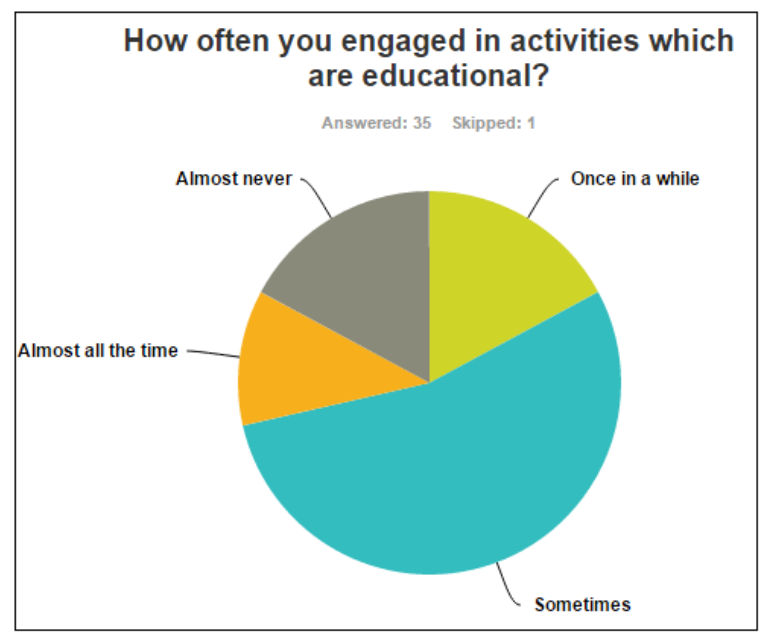

Fig. 18. Pie chart of activities in education

Fig. 18 shows that there were $17.14 \%$ respondents engaged in activities which are educational once in a while, 54.29\% respondents engaged sometimes, $11.43 \%$ respondents engaged almost all the time, and $17.14 \%$ respondents who engaged almost never in educational activities.

\section{4) Clear and understandable}

TABLE IV. SHOWING THE BASIC STATISTICS OF THE IS CLEAR AND UNDERSTANDABLE

\begin{tabular}{|l|l|l|l|l|l|}
\hline \multirow{3}{*}{ Sr.no } & \multicolumn{5}{|c|}{ basic statistics } \\
\cline { 2 - 6 } & Minimum & maximum & Median & mean & standard deviation \\
\cline { 2 - 6 } 1. & 1.00 & 4.00 & 2.00 & 2.29 & 0.94 \\
\hline
\end{tabular}

The minimum and maximum of 1 and 4 show that there was one response in the uppermost answer (i.e. strongly disagree) and three responses in the lower most answer (i.e. strongly agree). The median of 3.00 (higher than the 2.72 mean) shows that there were more respondents who were agreed than the respondents who were strongly agreed. A mean of 2.72 represents that the overall respondents came in somewhere between agreed or strongly agreed that the interaction with this application is clear and understandable. Ultimately, the standard deviation is 0.65 which shows the progress, dispersion, and variation of your responses.

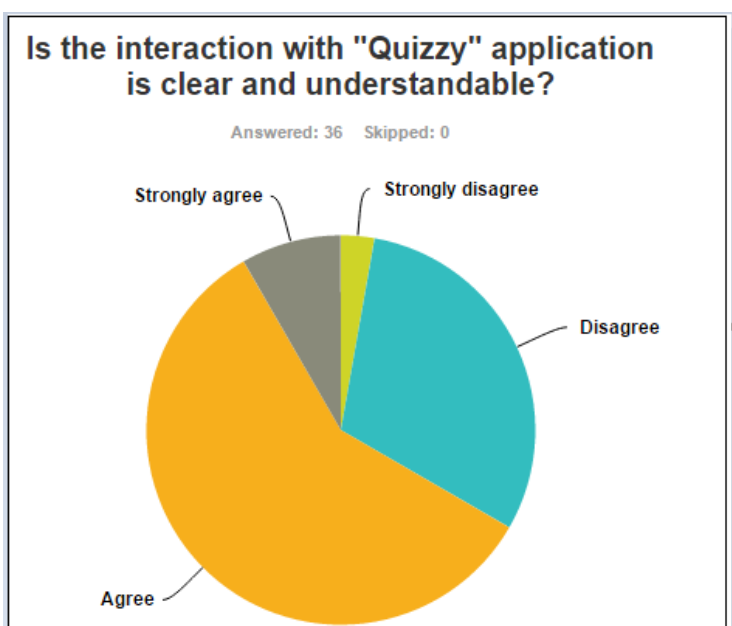

Fig. 19. Pie chart showing respondents calling the application as clearly understandable

Fig. 19 shows that $2.78 \%$ respondents strongly disagreed with the statement, $30.56 \%$ respondents disagreed, $58.33 \%$ respondents agreed, and $8.33 \%$ respondents strongly agreed with the statement that the interaction with "Quizzes" application is clear and understandable.

\section{5) Effort and practice required}

TABLE V. SHOWING THE BASIC STATISTICS OF THE EFFORT AND PRACTICE REQUIRED

\begin{tabular}{|l|l|l|l|l|l|}
\hline \multirow{3}{*}{ Sr.no } & \multicolumn{5}{|c|}{ basic statistics } \\
\cline { 2 - 6 } & Minimum & maximum & Median & mean & standard deviation \\
\cline { 2 - 6 } 1. & 1.00 & 4.00 & 3.00 & 2.72 & 0.65 \\
\hline
\end{tabular}

The minimum and maximum of 1 and 4 show that there were four responses in the uppermost answer (i.e. strongly disagree) and one response in the lower most answer (i.e. strongly agree). The median of 2.00 (lower than the 2.26 mean) shows that there were more respondents who disagreed than the respondents who agreed. A mean of 2.26 represents the overall respondents came in somewhere between disagreeing and agree. Here the standard deviation is 0.69 which shows the progress, dispersion, and variation of responses. 


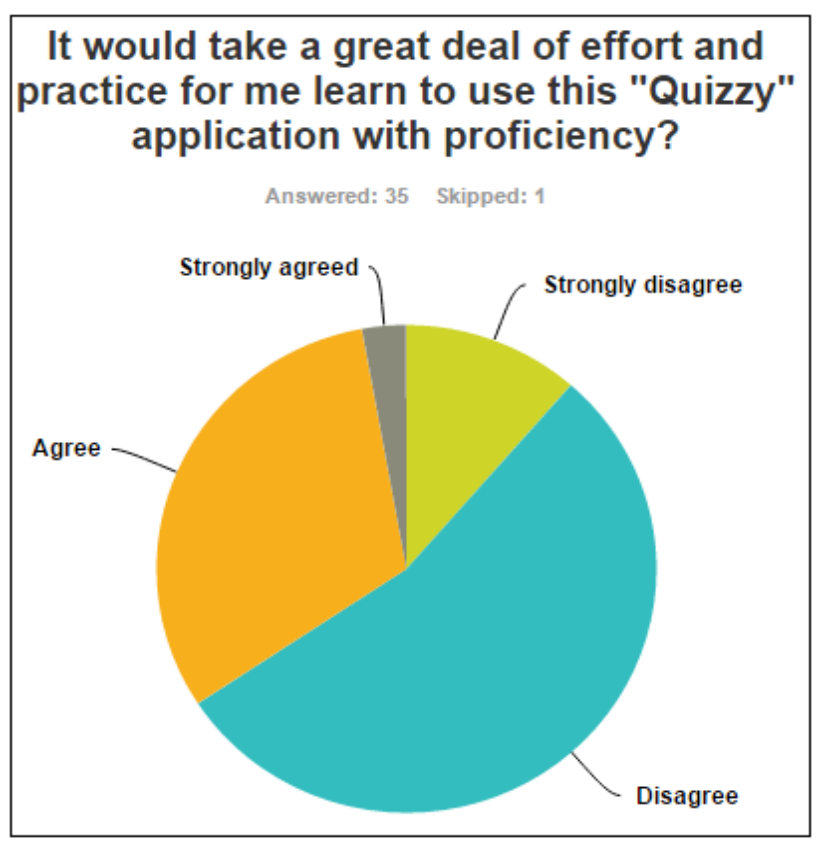

Fig. 20. Pie chart showing the effort and practice required

Fig. 20 shows that $11.43 \%$ respondents strongly disagreed with the statement, $54.29 \%$ respondents disagreed, $31.43 \%$ respondents agreed, and $2.86 \%$ respondents strongly agreed with the statement that it would take a great deal of effort and practice for them to learn to use this "Quizzes" application with proficiency.

\section{6) Satisfactory user interface design application or not}

TABLE VI. ShOWING THE BASIC STATISTICS OF SATISFactory USER INTERFACE DESIGN

\begin{tabular}{|l|l|l|l|l|l|}
\hline \multirow{3}{*}{ Sr.no } & \multicolumn{5}{|c|}{ basic statistics } \\
\cline { 2 - 6 } & Minimum & maximum & Median & mean & standard deviation \\
\cline { 2 - 6 } 1. & 1.00 & 4.00 & 2.00 & 2.26 & 0.69 \\
\hline
\end{tabular}

The minimum and maximum of 1 and 2 show that there were 23 responses in the uppermost answer (i.e. yes) and 13 responses in the lower most answer (i.e. No). The median of 1.00 (lower than the 1.36 mean) shows that there were more respondents who said Yes than the respondents who said No. A mean of 1.36 represents the overall respondents came in somewhere between Yes and No. Ultimately, the standard deviation is 0.48 which shows the progress, dispersion, and variation of your responses

Fig. 21 shows that $63.89 \%$ respondents replied Yes that they are satisfied with the design of this application's user interface and $36.11 \%$ respondents replied No that they are not satisfied with the design of this application's user interface.

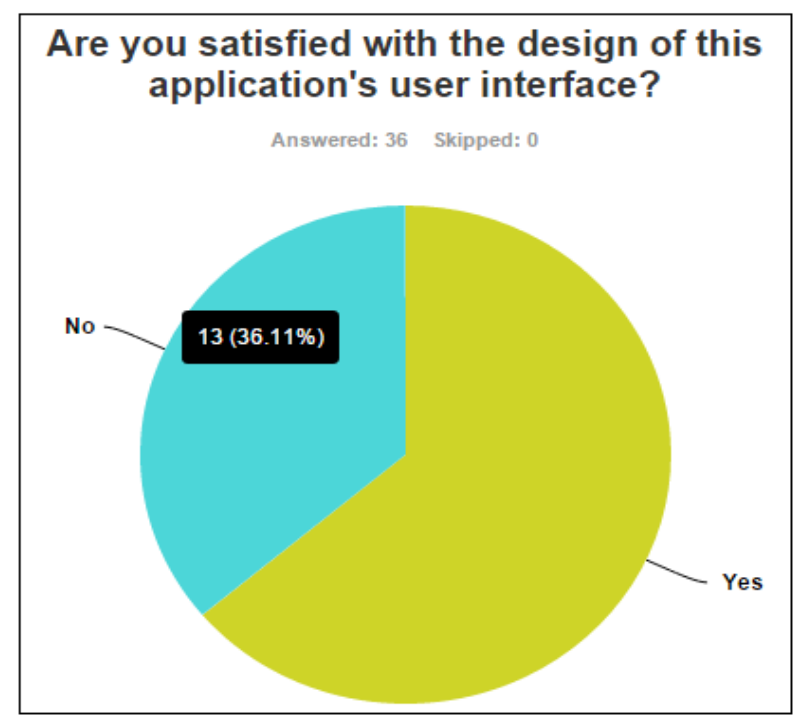

Fig. 21. Pie chart of user satisfaction for the design of the application

7) Why use Quizzes?

TABLE VII. SHOWING THE BASIC STATISTIC OF THE REASON FOR USING THE QUIZZY APPLICATION

\begin{tabular}{|l|l|l|l|l|l|}
\hline \multirow{3}{*}{ Sr.no } & \multicolumn{5}{|c|}{ basic statistics } \\
\cline { 2 - 6 } & Minimum & maximum & Median & mean & standard deviation \\
\cline { 2 - 6 } 1. & 1.00 & 4.00 & 4.00 & 3.11 & 1.07 \\
\hline
\end{tabular}

The minimum and maximum of 1 and 4 show that there were three responses in the uppermost answer (i.e. For time pass) and 20 responses in the lower most answer (i.e. For education and entertainment purpose). The median of 4.00 (higher than the 3.11 mean) shows that there were more respondents who used this application for education and entertainment purpose than the respondents who used this application for education purpose only.

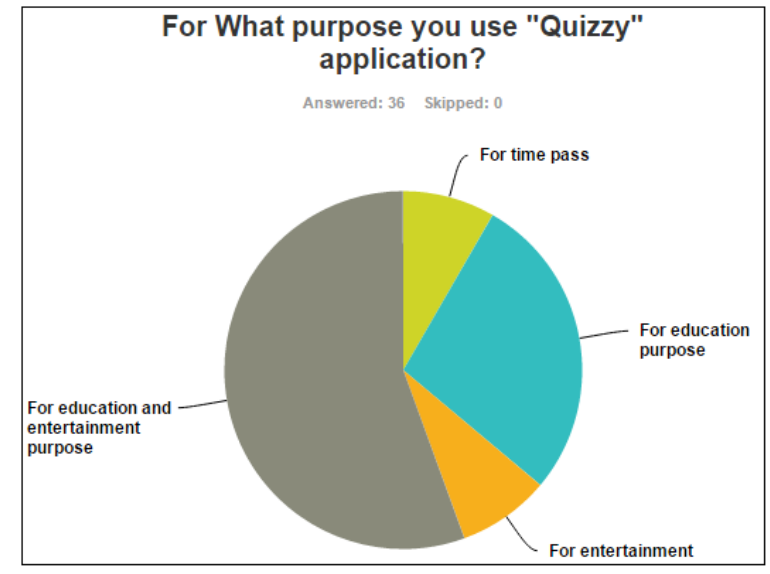

Fig. 22. Pie chart showing the purpose of Quizzes application 
A mean of 3.11 represents the overall respondents came in somewhere between for education purpose and for education and entertainment purpose. Ultimately, the standard deviation is 1.07 which shows the progress, dispersion, and variation of your responses.

The above Fig. 22 shows that $8.33 \%$ respondents used this application for time pass, $27.78 \%$ respondents used it for education purpose, $8.33 \%$ respondents used for entertainment, and $55.56 \%$ respondents used this application for education and entertainment purpose.

\section{8) Satisfied with Quizzes' ability or not}

TABLE VIII. Showing The Basic Statistic of ApPlication Ability

\begin{tabular}{|l|l|l|l|l|l|}
\hline \multirow{3}{*}{ Sr.no } & \multicolumn{5}{|c|}{ basic statistics } \\
\cline { 2 - 6 } & Minimum & maximum & Median & mean & standard deviation \\
\cline { 2 - 6 } 1. & 1.00 & 4.00 & 3.00 & 2.74 & 0.65 \\
\hline
\end{tabular}

The minimum and maximum of 1 and 4 show that there was one response in the uppermost answer (i.e.Very unsatisfied) and three responses in the lower most answer (i.e. Very satisfied). The median of 3.00 (higher than the 2.74 mean) shows that there were more respondents who were satisfied than the respondents who were unsatisfied. A mean of 2.74 represents the overall respondents came in somewhere between unsatisfied and satisfied. Ultimately, the standard deviation is 0.65 which shows the progress, dispersion, and variation of your responses

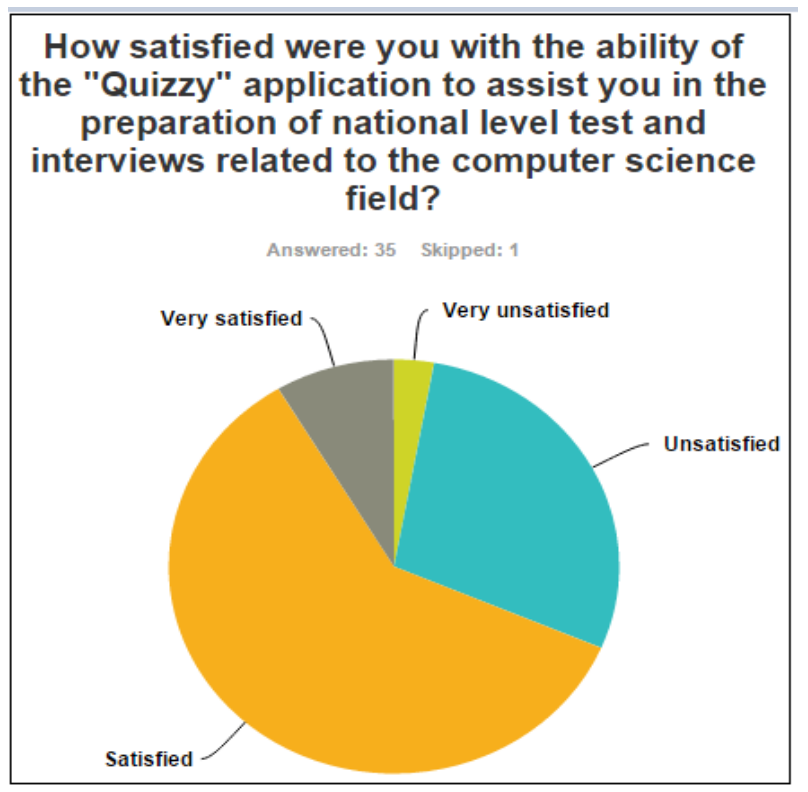

Fig. 23. Pie chart showing the ability of Quizzes application

Fig. 23 shows that $2.86 \%$ respondents were very unsatisfied, $28.57 \%$ respondents were unsatisfied, $60.00 \%$ respondents were satisfied and $8.57 \%$ respondents were very satisfied with the ability of the "Quizzes" application to assist them in the preparation of national level tests and interviews related to the computer science field.

\section{Will you recommend this application or not?}

TABLE IX. SHOWING THE BASIC STATISTICS OF RECOMMENDING THE APPLICATION OR NOT

\begin{tabular}{|l|l|l|l|l|l|}
\hline \multirow{3}{*}{ Sr.no } & \multicolumn{5}{|c|}{ basic statistics } \\
\cline { 2 - 6 } & Minimum & maximum & Median & mean & standard deviation \\
\cline { 2 - 6 } 1. & 1.00 & 2.00 & 1.00 & 1.31 & 0.46 \\
\hline
\end{tabular}

The minimum and maximum of 1 and 2 show that there were 25 responses in the uppermost answer (i.e. I will recommend) and 11 responses in the lower most answer (i.e. I will never recommend). The median of 1.00 ( lower than the 1.31 mean) shows that there were more respondents who will likely to recommend this application to others than the respondents who will never recommend this application. A mean of 1.31 represents the overall respondents came in somewhere between who will likely to recommend and who will never recommend. Ultimately, the standard deviation is 0.46 which shows the progress, dispersion and variation of your responses.

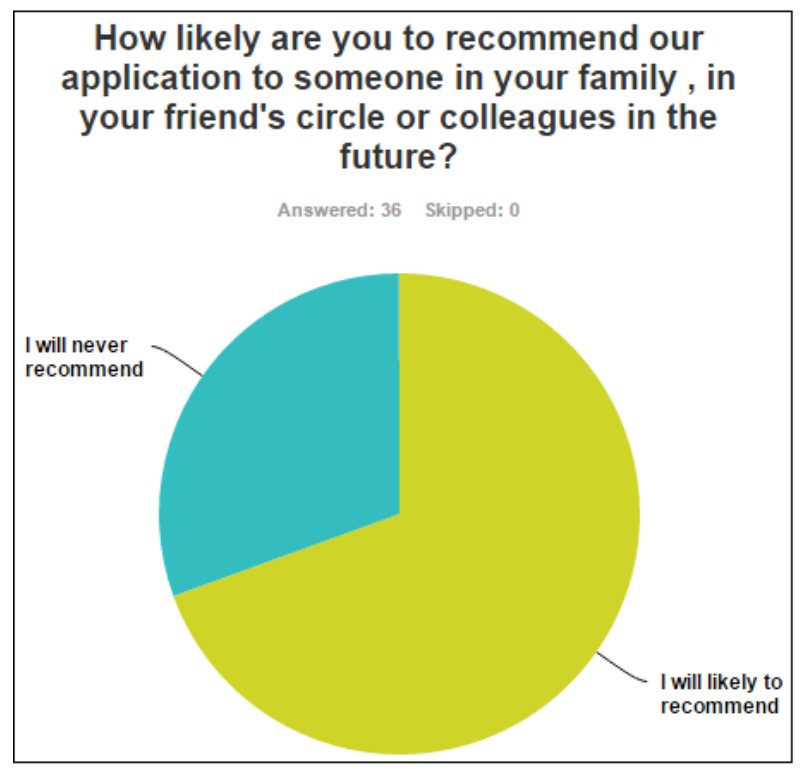

Fig. 24. Pie chart showing the recommendation of the application.

Fig. 24 shows that $69.44 \%$ respondents were likely to recommend this application and $30.56 \%$ respondents will never recommend this application to others.

\section{REFERENCES}

[1] Byers, J.A. 1999.” Interactive Learning Using Expert System Quizzes on the Internet. Educational Media International" 36:191-194. Available at: http://www.chemical-ecology.net/papers/expert.htm/ last accessed, 22 Nov, 2015

[2] Available at: http://www.wcrl.ars.usda.gov/download/itquiz.zip/ last accessed, 22 Nov 2015

[3] Available at: http://wcrl.ars.usda.gov/cec/udt/exam-f.htm/ last accessed, 22 Nov 2015

[4] Systems analysis and Design methods Available at: http://highered.mheducation.com/sites/0073052337/student_view0/chapt er2/multiple_choice_quiz.html/ last accessed, 22 Nov 2015 
[5] Quiz

Hub

Available at: http://quizhub.com/quiz/quizhub.cfm/ last accessed, 22 Nov 2015

[6] Jagran Josh (Simplifying Test

Prep) Available at: http://www.jagranjosh.com/articles/computer-generalknowledge-quiz-1315979215-1/ last accessed, 24 Nov 2015

[7] EDU Zip TheKnowledge

Hub Available at: http://www.eduzip.com/category/computer-science/ last accessed, 24 Nov 2015

[8] Tree Available at:http://www.treeknox.com/gk/gk/computerquiz/24 Nov 2015

[9] Available at: https://play.google.com/store?hl=en/ last accessed, 24 Nov 2015

[10] Available at: https://play.google.com/store?hl=en/ last accessed, 24 Nov 2015

[11] Saqib SM, Asghar MZ, Ahmad S, Ahmad B, Jan MA. "Framework for Customized-SOA Projects". International Journal of Computer Science and Information Security. 2011 May 1;9(5):240.
[12] Asghar D, Zubair M, Asghar MJ. "Expert System For Online Diagnosis of Red-Eye Diseases". International Journal of Computer Science \& Emerging Technologies (IJCSET). 2010;1(2):35-9.

[13] Saqib SM, Jan MA, Ahmad B, Ahmad S, Asghar MZ. "Custom Software under the Shade of Cloud Computing". International Journal of Computer Science and Information Security. 2011 May 1;9(5):219.

[14] Hussain S, Asghar MZ, Ahmad B, Ahmad S. "A Step towards Software Corrective Maintenance Using RCM model". arXiv preprint arXiv:0909.0732. 2009 Sep 3.

[15] Rashid A, Zubair MZ. An Intelligent Agent for a Vacuum Cleaner. International Journal of Digital Content Technology and its Applications. 2009;3(2):143-6.

[16] Asghar MZ, Ahmad S, Marwat A, Kundi FM. "Sentiment Analysis on YouTube: A Brief Survey". arXiv preprint arXiv:1511.09142. 2015 Nov 30 .

[17] Asghar, Dr, Muhammad Zubair, and Dr Ahmad. "A Review of Location Technologies for Wireless Mobile Location-Based Services." Journal of American Science 10.7 (2014): 110-118. 